\title{
Thiamine status in inherited degenerative ataxias
}

\author{
O L Pedraza, M I Botez
}

Neurology Service, Hôtel-Dieu de Montréal, 3840 StUrbain Street, Montreal, Quebec, Canada H2W 1T8 O L Pedraza M I Botez

Correspondence to: Dr Botez Received 30 January 1991 and in revised form 20 May 1991.

Accepted 13 June 1991

\begin{abstract}
Blood thiamine levels in ataxia patients were studied. No significant differences were found between 30 patients with Friedreich's ataxia and 29 patients with olivopontocerebellar atrophy (OPCA) compared with control subjects. Both OPCA and Friedreich's ataxia patients presented significantly lower cerebrospinal fluid thiamine levels than their controls $(p<0.001$ and $p<0.04$ respectively). These results, discussed in terms of the high degree of cerebellar atrophy on CT scans in OPCA $v$ Friedreich's ataxia patients, seem to correlate with cerebellar thiamine turnover and content.
\end{abstract}

Despite extensive experimental data reported in the literature, there are few studies on the thiamine content of human cerebrospinal fluid (CSF). ${ }^{2}$ Low blood thiamine levels have been found in some Friedreich's ataxia patients, ${ }^{3}$ whereas in 10 subjects with four forms of ataxias of degenerative origin, thiamine and thiamine monophosphate were significantly reduced in cerebrospinal fluid (CSF) but not in plasma. ${ }^{4}$ We report on blood and CSF thiamine levels in patients with olivopontocerebellar atrophy (OPCA) and Friedreich's ataxia and normal controls.

\section{Patients and methods}

We studied 30 patients with Friedreich's ataxia and 29 with OPCA who were hospitalised. Their blood and CSF thiamine levels were compared with two respective groups of normal subjects (see table). There were therefore two control groups matched for age with the patient groups. All patients and controls underwent dietary assessment before inclusion in the study. The exclusion criteria were diabetes; alcoholism; dietary deficiency; and vitamin supplement intake in the four months before the study. The patients with Friedreich's ataxia fulfilled all the clinical and genetic criteria of Harding; ; patients with known cardiomyopathy were excluded because they had also served in an amantadine clinical trial. Amantadine has to be avoided in cardiomyopathies. For this reason only two of these 30 patients had cardiomyopathies diagnosed during hospitalisation; 23 were confined to a wheelchair whereas seven moved with a walker. The OPCA patients (14 men and 15 women) were diagnosed and classified according to the clinical and genetic criteria of Huang and Plaitakis. ${ }^{6}$ Three of them had a dominant form of Menzel type OPCA, 11 had a dominant form with slow saccade eye movements, seven had a recessive form, whereas the remaining eight had sporadic forms. Six out of these 29 patients were confined to a wheelchair.

The control groups underwent spinal taps for myelograms carried out exclusively for lumbar disc herniations; the same exclusion criteria applied as for the patient groups. Before the spinal taps, all patients and controls signed an informed consent form as required by the ethics committee of the hospital. CT scans assessed the degree and severity of brain atrophy with special attention to cerebellar atrophy. ${ }^{78}$ Samples of CSF were collected by lumbar puncture between 8.30 and 9.30 am after 4-5 days of hospital diet; blood samples were taken before the lumbar puncture. Whole blood and CSF thiamine were always assayed in duplicate by a previously described microbiological method, with Lactobacillus fermenti. ${ }^{9}$ Lumbar punctures were not repeated in the same patients. Because of some variability in the results, the Mann-Whitney U test was used for group comparisons.

\section{Results}

Cerebellar atrophy was absent in eight, mild in sixteen and moderate in six of the 30 Friedreich's ataxia patients; in contrast, all 29 OPCA patients had different degrees of cerebellar atrophy: mild eight, moderate eleven, and severe ten. Blood thiamine levels were not significantly different between the two patient groups compared with their respective controls but CSF thiamine values were low in OPCA

Table Demographic data and thiamine determinations

\begin{tabular}{|c|c|c|c|c|c|c|c|c|c|c|}
\hline Variable & $\begin{array}{l}\text { Friedreich's } \\
\text { ataxia patients } \\
(14 W, 16 M)\end{array}$ & $v$ & $\begin{array}{l}\text { Controls } \\
\text { (10W, } 8 M)\end{array}$ & $Z$ & $p$ Value $e^{\star}$ & $\begin{array}{l}\text { OPCA patients } \\
(15 \mathrm{~W}, 14 \mathrm{M})\end{array}$ & $v$ & $\begin{array}{l}\text { Controls } \\
(13 W, 19 M)\end{array}$ & $Z$ & p Value \\
\hline $\begin{array}{l}\text { Mean (SD) age (years) } \\
\text { Mean (SD) duration of disease (years) } \\
\text { Mean (SD) blood thiamine (ng/ml) } \\
\text { Mean (SD) CSF thiamine (ng/ml) }\end{array}$ & $\begin{array}{l}29 \cdot 1(6 \cdot 5) \\
18 \cdot 1(6 \cdot 3) \\
49 \cdot 3(17 \cdot 7) \\
30 \cdot 6(12 \cdot 3)\end{array}$ & & $\begin{array}{l}31 \cdot 3(6 \cdot 8) \\
53 \cdot 3(18 \cdot 7) \\
37 \cdot 3(14 \cdot 4)\end{array}$ & $\begin{array}{l}-1 \cdot 13 \\
-1 \cdot 30 \\
-2 \cdot 09\end{array}$ & $\begin{array}{l}\text { ns } \\
\text { ns } \\
0 \cdot 04\end{array}$ & $\begin{array}{l}47 \cdot 6(13 \cdot 3) \\
13 \cdot 7(7 \cdot 4) \\
46 \cdot 3(13 \cdot 6) \\
23 \cdot 9(10 \cdot 9)\end{array}$ & & $\begin{array}{l}43 \cdot 9(15 \cdot 2) \\
51 \cdot 5(17 \cdot 1) \\
35 \cdot 4(12 \cdot 3)\end{array}$ & $\begin{array}{l}-1.43 \\
-1.82 \\
-4.02\end{array}$ & $\begin{array}{l}\text { ns } \\
\text { ns } \\
0 \cdot 001\end{array}$ \\
\hline
\end{tabular}

*Mann-Whitney U test. 
and Friedreich's ataxia patients (table). In an additional group of three patients with Charlevoix-Saguenay spastic recessive ataxia, ${ }^{10}$ CSF thiamine values were very low (mean (SD) $14.6(4 \cdot 2) \mathrm{ng} / \mathrm{ml}$ ) despite normal blood thiamine levels.

\section{Discussion}

The observed discrepancy between normal blood thiamine values and low CSF thiamine levels agrees with our previous findings in epileptic patients with cerebellar atrophies compared with those with normal CT scans $^{9}$ as well as with Poloni and Patrini's data ${ }^{4}$ on a small number of ataxic patients. The normal CSF values in the present study are higher than those in untreated epileptic patients reported in a previous investigation. This difference may be due to cerebellar atrophy being encountered in about $15 \%$ of cases of untreated epilepsy ${ }^{81}$; however, atrophy is more common and more severe in chronically treated epileptics. In our earlier study of 35 untreated epileptics, seven displayed mild or moderate cerebellar atrophy, and this could account for the difference because untreated epileptics should not be considered as normal controls; their CSF thiamine values were considered to be similar to control values only for the other epileptic groups. The low CSF thiamine values in our patients could not be explained by a deficient diet because all patients and controls had normal dietary assessments. The cerebellum, medulla, and pons in rats have the highest thiamine turnover rate in the CNS, ${ }^{12}$ the most obvious thiamine depletion occurring after deprivation, ${ }^{13}$ and these are the first regions to develop lesions. ${ }^{1415}$ The olivary nucleus, pontine nuclei, decussation of the superior cerebellar peduncles, and dentate nucleus are highly sensitive to thiamine deficiency in monkeys. ${ }^{16}$

Thiamine is transported across the bloodbrain barrier by two separate mechanisms: one saturable (probably carrier-mediated), which accounts for $91 \%$ (cerebral cortex) to $96 \%$ (cerebellum) of the total transport of labelled thiamine at physiological plasma levels, and the other non-saturable (4-9)..$^{16}$ If anything interferes with the carrier-mediated component of thiamine transport across the bloodbrain barrier, the supply of the vitamin in the brain will become inadequate. ${ }^{17} 18$

We propose two mechanisms to explain the low CSF thiamine levels in our patients in the absence of a blood thiamine deficiency. A primary mechanism located in the cerebellum itself - that is, cerebellar atrophy-may induce a lowering of brain and CSF thiamine because the cerebellum has the highest content and turnover of the vitamin among all other nervous structures ${ }^{12}$; the severe cerebellar atrophy encountered in OPCA patients could explain their low CSF thiamine levels whereas in Friedreich's ataxia patients in whom cerebellar atrophy is less pronounced, CSF thiamine levels are relatively higher; the low CSF thiamine values recorded in patients with Charlevoix-Saguenay spastic recessive ataxia may be attributed to their massive vermal atrophy. ${ }^{10}$ A secondary mechanism-that is, cerebellar and brainstem atrophy-may decrease the vascular surface, diminishing saturable transfer of the vitamin across the blood-brain barrier and reducing CSF thiamine levels.

This work was supported by grants from the Canadian Association of Friedreich's Ataxia and Fonds de la recherche en santé du Québec (FRSQ). Mr Marc Dumont, performed the statistical analysis. Thanks are due also to Mrs Ana De Oliveira for her technical assistance and Mrs Michèle Mathieu for her secretarial work.

1 Haas RH. Thiamine and the brain. A review. Ann Rev Nutr 1988;8:483-515.

2 Botez MI, Young SN, Bachevalier J, Gauthier S. Thiamine deficiency and cerebrospinal fluid 5-hydroxyindoleacetic acid: a preliminary study. J Neurol Neurosurg Psychiatry acid: a prelimina

3 Purkiss P, Baraitser M, Borud O, Chalmers RA. Biochemical urkiss $\mathrm{P}$, Baraitser M, Borud O, Chalmers RA. Biochemical
and clinical studies of Friedreich's ataxia. J Neurol and clinical studies of Friedreich's

4 Poloni M, Patrini C. Cerebrospinal fluid, plasma and erythrocyte thiamine levels in ataxias of degenerative origin. Ital J Neurol Sci 1984;suppl 4:141-3.

5 Harding AE. Friedreich's ataxia: a clinical and genetic study of 90 families with an analysis of early diagnostic criteria and intrafamilial clustering of clinical features. Brain 1981;104:589-620.

6 Huang YP, Plaitakis A. Morphological changes of olivopontocerebellar atrophy in computed tomography and comments on its pathogenesis. In: Duvoisin RC, Plaitakis A, eds. The olivopontocerebellar atrophies. New York: A, eds. The olivopontocerebel

7 Koller WC, Glatt SL, Perlik S, Huckman MS, Fox JH. Cerebellar atrophy demonstrated by computed tomograCerebellar atrophy demonstrated

8 Botez MI, Attig E, Vézina JL. Cerebellar atrophy in epileptic patients. Can J Neurol Sci 1988;15:299-303.

9 Botez MI, Young SN. Effects of anticonvulsivant treatment and low levels of folate and thiamine on amine metabolites in cerebrospinal fluid. Brain 1991;114:333-48.

10 Bouchard JP, Barbeau A, Bouchard R, Bouchard RW. Autosomal recessive spastic ataxia of CharlevoixSaguenay. Can J Neurol Sci 1978;5:61-9.

11 Dam M. Chronic toxicity of antiepileptic drugs with respect to cerebellar and motor function. In: Oxley J, Janz D, Meinhardi $\mathrm{H}$, eds. Chronic toxicity of antiepileptic drugs, New York: Raven Press 1983:223-8.

12 Rindi G, Patrini C, Comincioli V, Reggiani C. Thiamine content and turnover rates of some rat nervous regions, content and turnover rates of some rat nervous regions, 181:369-80.

13 Pincus JM, Grove Y. Distribution of thiamine phosphate esters in normal and thiamine deficient brain. Exp Neurol esters in normal

14 Collins GH, Converse WK. Cerebellar degeneration in thiamine deficient rats. Am J Pathol 1967;58:219-33.

15 Robertson DM, Wasan SM, Skinner DB. Ultrastructural changes of features of early brainstem lesions in thiamine deficient rats. Am J Pathol 1968;52:1081-7.

16 Witt ED, Goldman-Rakic P. Intermittent thiamine deficiency in the rhesus monkey. I. Progression of neurological signs and neuroanatomical lesions. Ann Neurol 1983;13:376-95.

17 Greenwood J, Love ER, Pratt OE. Kinetics of thiamine transport across the blood-brain barrier in the rat. $J$ Physiol 1982;327:95-103.

18 Reggiani C, Patrini C, Rindi G. Nervous tissue thiamine metabolism in vivo. I. Transport of thiamine and thiamine monophosphate from plasma to different brain regions of the rat. Brain Res 1984;293:319-27. 